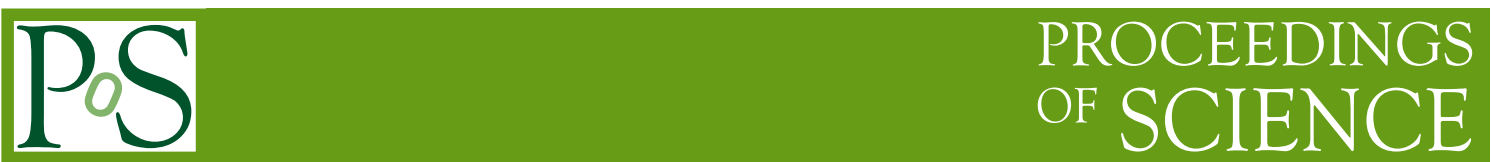

\title{
An Attempt of Construction for the Grassmann Numbers
}

\section{Ricardo Bentin}

Departamento Ciências Exatas e Tecnológicas - DCET

Universidade Estadual de Santa Cruz.

Rodovía Ilhéus-Itabuna km 16 s/n, CEP 45662-000, Ilhéus-BA, Brazil.

E-mail: rbentin@uesc.br

\begin{abstract}
We will pursue a way of building up an algebraic structure that involves, in a mathematical abstract way, the well known Grassmann variables. The problem arises when we tried to understand the grassmannian polynomial expansion on the scope of ring theory. The formalization of this kind of variables and its properties will help us to have a better idea of some algebraic structures and the way they are implemented in models concerning theoretical physics.
\end{abstract}

Fifth International Conference on Mathematical Methods in Physics - IC2006

April 24-28 2006

Centro Brasilerio de Pesquisas Fisicas, Rio de Janeiro, Brazil 


\section{Introduction.}

Let's consider this:

Definition 1 (Grassmann Variable) Let $\mathbb{G}$ be a non-empty subset and let $(\cdot)$ and $(+)$ be two binary operations on this set such as:

$$
\mathbb{G}=\left\{\theta_{i} / \theta_{i} \cdot \theta_{j}+\theta_{i} \cdot \theta_{j}=0\right\}
$$

where 0 is the additive identity of the complex ring. We define the set $\mathbb{G}$ as being the set of the Grassmann variables.

With this definition we are starting a study that will try to formalize a tool used in mathematicalphysics [1]. Without going throughout philosophical aspects, we can cite as an example the case of the polynomials defined over a field $K$ when they are written in its usual form in terms of the variable $x$,

$$
p(x)=a_{0}+a_{1} x+\cdots+a_{n} x^{n},
$$

the set of these polynomials describe an structure called Euclidean Domain [2] and the notation used is $p(x) \in K[x]$. But this notation hides an important fact: we are actually working with an infinite sequence of elements that belongs to the same algebraic structure: the field $K$.

But the Definition 1 is not at all completely consistent, since it let us some questions without answer or in some sense ambiguity answers. For example, working with the set $\mathbb{G}$, will it be an extension of some another field (such as the field $\mathbb{R}$ field or the $\mathbb{C}$ field)? Or may this kind of variables can be expressed in terms of known numerical sets? And what about the binary operations over the set $\mathbb{G}$, how can we define them? The following section will contain a detailed exposition of the problem from the point of view of the the mathematical rigor and under the scope of the theoretical physics, when this kind of variables are used in a polynomial expansion.

\section{The Problem.}

Works in theoretical physics consider that one fundamental particle in nature obey just one of the two possible statistics [3]:

- Quantum Fermi-Dirac statistics, and

- Quantum Bose-Einstein statistics.

This means that, in a mathematical language, we have two equivalence classes over the set of the fundamental particles: the set of fermions and the set of bosons. To obtain a mathematical description of the quantum fermionic fields it is been used the Clifford Algebra (a "slightly" variant of Grassmann Algebra) [3, 4]. This statistical classification let us to arrive to the very important concept of supersymmetry in which the number of fermions is equal to the number of bosons. But when this concept is transcribed to the mathematical language, is mandatory the use of a new kind of variables to obtain a consistent supersymmetric space or say it in a short way: the superspace. This superspace makes use of the definition of Grassmann variable and it is associated to the theoretical 
physics in a bijective way: one fermionic coordinate is represented by one Grassmann variable. This kind of construction is well known in mathematical-physics theories that involve supersymmetry [4]. It is easy to see that Definition 1 implies $\theta^{2}=0$, then we can build up polynomials of the form:

$$
p(\theta)=a_{0}+a_{1} \theta
$$

where $a_{0} \in \mathbb{C}$ and $a_{1}, \theta \in \mathbb{G}$.

But a carefully observation allows us to see that there is a small inconsistency (from the mathematical point of view). To take account of that, let's remember how a polynomial is defined according the fundamentals of algebra:

Definition 2 (Polynomial) Let $\langle A,+, \cdot\rangle$ be $a$ ring and $a_{i} \in A$ where $i=0, \cdots, n$ and $n \in \mathbb{N}$. We define the finite sequence $\left\{a_{0}, \cdots, a_{n}\right\}$ of elements that belongs $A$ as being a polynomial of degree $n$ over the ring $A$.

But in the polynomial of equation (2.1) we have that $a_{0}$ is over the ring of the complex numbers but $a_{1}$ does not belong to that ring. $a_{1}$ is on the set $\mathbb{G}$ and we do not have an idea of what is the algebraic structure that $\mathbb{G}$ belongs to! In order to have $\mathbb{G}$ as a ring structure we must have two binary operations. But our starting definition lead us to think in a binary relation of the form $\mathbb{G} \times \mathbb{G} \mapsto \mathbb{C}$ that also restricts our idea of binary operation! In other words, we are in trouble when consider Definition 1 under a mathematical lens.

\section{Grassmann Variables and $2 \times 2$ Matrices.}

This section is motivated by the fact that $a_{0} \in \mathbb{C}$ and the well known fact of the isomorphism between the fields of the complex numbers and a subset $N$ of the real $2 \times 2$ matrices $\varphi: \mathbb{C} \mapsto N$, such as:

$$
a+b i \mapsto\left(\begin{array}{cc}
a & -b \\
b & a
\end{array}\right) .
$$

The main idea of this section is to search for some second order matrix representation that obeys Definition 1 with the condition that the operation of two Grassmannian variables lead us into a complex number, which can also be represented as a second order matrix. Another motivation is the fact that in general the product of two matrices is not commutative and if we find an appropriate matrix representation, the binaries operations over the set $\mathbb{G}$ will be automatically defined as the operations of addition and multiplication of second order matrices. The following result will help us in our analyze.

Lemma 1. Let $a, b \in \mathbb{R}$ both different from zero, the $2 \times 2$ matrix that have the form $\left(\begin{array}{cc}a b & b^{2} \\ -a^{2} & -a b\end{array}\right)$ fulfills Definition 1 but is unique unless real multiples of it.

Proof It is easy to see that the given matrix fulfills the definition of the Grassmann variables algebra, then let

$$
\left(\begin{array}{cc}
c d & d^{2} \\
-c^{2} & -c d
\end{array}\right)
$$


be another matrix that obeys the same Definition, in this case these two matrices must also obey the same definition, but this implies the condition $(a d-b c)^{2}=0$, i. e., $a d=b c \doteq w \in \mathbb{R}$ that allows us to write $d=w / a$ e $c=w / b$, then the second matrix will be written as

$$
\frac{w^{2}}{(a b)^{2}}\left(\begin{array}{cc}
a b & b^{2} \\
-a^{2} & -a b
\end{array}\right) \text {. }
$$

This Lemma allow us to conclude that is not possible to construct a linearly independent set of second order matrices that respects the definition of Grassmann variables and also when multiplied the result is a non-null complex number associated to another second order matrix.

\section{Grassmann Variables and the Complex Numbers.}

Since it is impossible to build a set of $2 \times 2$ matrices that can be represented as the Grassmann variables of the Definition 1, let's try another possibility, now working with the idea of binary operations among the Grassmann variables. For this motive, we will give the following definitions,

Definition 3 (Odd Complex Function) Let $F(z): \mathbb{C} \mapsto \mathbb{C}$ a complex number application over the field $\mathbb{C}$, we define this function as an odd complex function if, and only if $F(z)=-F(-z)$ for all $z \in \mathbb{C}$.

Definition 4 (Binary Operation $*$ ) Let $\theta_{1}=z_{1}$ and $\theta_{2}=z_{2}$ such us $z_{1}, z_{2} \in \mathbb{C}$ two elements of the complex numbers field, we define the Grassmann multiplication $(*)$ over $\mathbb{C}$ such us $\theta_{1} * \theta_{2}=$ $F\left(z_{1}-z_{2}\right)$ where $F(z)$ is and odd complex function.

It is important to point out about this definition that the use of the $\theta$ variables only represent a label, since they are in fact elements of the complex number set, but now with the operation $*$ defined do not form a group, but a grupoid.

With this last Definition 4 of binary operation $*$ we can shown that the Definition 1 is also valid.

But here we have to make some observations about it since the polynomial (2.1) has elements of the Grassmann type and also of the complex number type. Then it is necessary to define which binary operations can be used to relate them, i.e. $z R \theta$. In this case we have two choices: $z \cdot \theta$ or $z * \theta$, taking in account physical considerations the suitable choose between $z$ and $\theta$ will be $(\cdot)$.

The use of an odd function when the Definition 4 was given have some interesting consequences on the scope of the mathematical-physics, since the non-commutative geometry [5] makes use of

$$
\left[\theta_{i}, \theta_{j}\right]=i \Omega_{i j}
$$

Where $\left[\theta_{i}, \theta_{j}\right] \equiv \theta_{i} * \theta_{j}-\theta_{j} * \theta_{i}$ represents the commutator, that when mixed with the definition of the $*$ product and the fact that $F(z)$ is odd lead us to the identification

$$
\Omega_{i j}=-i 2 F\left(z_{i}-z_{j}\right)
$$

that written in the form of the product $\star$, is known as the Moyal product [5] for two functions:

$$
f(x) \star g(y)=\left.e^{F\left(z_{i}-z_{j}\right) \partial_{x_{i}} \partial_{y_{j}}} f(x) g(y)\right|_{x=y},
$$


or using the Definition 4

$$
f(x) \star g(y)=\left.e^{\theta_{i} \theta_{j} \partial_{x_{i}} \partial_{y_{j}}} f(x) g(y)\right|_{x=y} .
$$

Now the question will be about the form that the odd function of the Definition 4 must have. At this time we do not have an answer, but surely we can say that the form of this odd function $F(z)$ must be such as it will generate an interesting algebraic structure and of course also obey criteria that belong to the physical theories.

\section{Conclusion.}

The construction of Grassmann numbers as was done in this work shows that the behavior of Definition 1 in terms of two binary operations over the field $\mathbb{C}$ and the $2 \times 2$ matrices lead us to contradictions since we were interested in preserve both, the definition of the Grassmann variables and the equation of the Grassmannian polynomial, at the same time.

This motivates us to make a new definition, now over the binary operations of the set $\mathbb{C}$ in order to be consistent with the initial definition of the Grassmann variable. This construction only makes a difference among the Grassmann and complex numbers just in the way they make use of the binary operation, in other words they represent the same set of numbers but they do not form the same algebraic structure. It means that there is nothing wrong if we now consider $\mathbb{G} \times \mathbb{G} \mapsto \mathbb{C}$ (fermionfermion), $\mathbb{C} \times \mathbb{G} \mapsto \mathbb{G}$ (boson-fermion) and $\mathbb{C} \times \mathbb{C} \mapsto \mathbb{C}$ (boson-boson) since we are working with the same set of numbers.

Discussions about this work with professor Lev Birbrair (Universidade Federal do Ceará) pointed us that this work could be related with the Operator Theory [6, 7].

\section{References}

[1] F.A. Berezin, Method of Second Quantization, Academic Press (1966).

D. Olive, P.C. West, and H. K. Moffatt (Editors), Duality and Supersymmetric Theories (Publications of the Newton Institute), Cambridge University Press (1999),

[2] L.H. Monteiro, Elementos de Álgebra, LTC, Rio de Janeiro (1978),

R. Dean, Elementos de Álgebra Abstrata, LTC, Rio de Janeiro, (1974),

[3] N.N. Bogoliubov and D.V. Shirkov, Quantum Fields, Benjamim/Cummings (1983).

[4] S.J. Gates, M.T. Grisaru, M. Rocek, and W. Siegel, Superspace, or One thousand and one lessons in supersymmetry, Front.Phys. 58 (1983),

M.B. Green, J.H. Schwarz, and E. Witten, Superstring Theory: Volume 2, Introduction (Cambridge Monographs on Mathematical Physics), Cambridge University Press, (1988),

[5] N. Seiberg, and E. Witten, String Theory and noncommutative geometry, JHEP 9909:032, (1999).

[6] J. Lepowsky, and H. Li, Introduction to vertex operator algebras and their representations. Progress in Mathematics, 227. Birkhäuser Boston, Inc., Boston, MA, (2004),

J. Sauvageot, A survey of operator algebras, Quantum probability communications, Vol. XII (Grenoble, 1998), 173-194, QP-PQ, XII, World Sci. Publishing, River Edge, NJ.

[7] B. Farb, and R.K. Dennis, Noncommutative Algebra, Springer-Verlag New York, (1983). 